MATEC Web of Conferences 22,02028 (2015)

DOI: $10.1051 /$ matec conf/ 20152202028

(C) Owned by the authors, published by EDP Sciences, 2015

\title{
Research on Predicting Drive Current of Shipborne Satcom Antenna
}

\author{
Jinping Kong*, Zhengfeng Xu \& Botao Wu \\ China Satellite Maritime Tracking and Control, Jiangyin, Jiangsu, China
}

\begin{abstract}
Predicting the effect of antenna wind load on servo system precisely is meaningful to ensure the safety of satcom antenna on operation, which can avoid overload operation. In this paper, the computational fluid dynamics is used to proceed numerical computation on the pressure distribution of the reflector and torque of drive shaft under different wind speed, windward angle and angle of pitch of the antenna. The simulation model is built under MATLAB/Simulink simulation environment, and the drive current of the antenna servo system is analyzed under wind load effect and ship swing. Then, a method of predicting drive current of antenna servo system according to the wind speed, wind direction and attitude of the antenna is concluded. And this method is verified by simulation at last.
\end{abstract}

Keywords: shipborne satcom antenna; wind load; CFD; MATLA/Simulink

\section{INTRODUCTION}

In the shipborne satcom, the motors drive the antenna to isolate the ship swing and compensate the position and ship heading change during sailing to make sure that the antenna always aims at the target satellite. For outdoor antenna, the wind load is the main load that must be considered, which not only can cause the transformation of the reflector and back frame, the feed displacement and pointing error, but also can apply the wind torque disturbance to the antenna servo system shaft and affect the tracking stabilization and accuracy of the antenna servo system. For the shipborne satcom antenna with complicated servo system, the influence of wind load on servo system is very huge. Hence calculating the wind load bearing by the antenna accurately and predicting the drive current are significant to assess whether the antenna servo system can operate normally and avoid the overload operation.

\section{CALCULATION OF ANTENNA WIND LOAD}

With the research of computational fluid dynamics and the fast development of computer hardware since 1990 s, numerical analysis on wind load is used in practical engineering projects continuously. In 1997, Selvam and others analyzed the Texas science research building by numerical analysis and the numerical simulation results which are accorded with the practical measurement results quite well [1]. In 2009, Du Qiang and Du Pingan from University of Electronic Science and Technology of China made numerical simulations on pressure distribution of rectangle flat antenna which has experimental results from wind tunnel [2], and also on wind load of vehicle-mounted radar antenna, and they obtained the features of an- tenna wind load [3]

All fluid in practical engineering projects such as the simple two-dimensional jet flow, wake flow, tunnel flow and boundary flow on a plate and the complex three-dimensional fluid, can be unstable when the Reynolds factor is larger than some value, and cause fierce changes of the flow pattern which is turned into the stochastic and chaos pattern. The fluid pattern whose speed and other fluid variables change in stochastic and chaotic way is called turbulence. Although turbulence obeys the Navier-Stokes [4] equation, the direct numerical simulation on the real-time movement of turbulence is limited by computer speed and capacity, even the modern computer cannot do the job. Due to this dilemma, people try to bring the turbulence model into the structure-complex turbulence simulation. In this paper, the standard $k-\varepsilon$ model which is the most widely-used and effective model is used and achieves impressive success in thin shear layer and back flow, and the model parameters adjustment is not needed with conditions' change.

\subsection{Computational model}

For most engineering projects, analysis of the turbulence pulsation details is not needed, and generally, only the influence of turbulence on time-mean flow is needed.

As shown in Figure 1, speed can be decomposed into the steady-state time-mean value $U$ and the pulsation $u^{\prime}(t)$, which is $u(t)=U+u^{\prime}(t)$. The general way is to assign features for turbulence with the statistics of time-mean value $(U, V, W, P$, and so on) and their pulsation $\left(u^{\prime}, v^{\prime}, w^{\prime}, p^{\prime}\right.$, and so on). And the turbulence simulation which is to directly compute the mean value and the influence of pulsation on mean value is done by simple approximate simulation.

In the standard $k-\varepsilon$ model, the $k$ and $\varepsilon$ are

*Corresponding author: kongjinping1981@163.com

This is an Open Access article distributed under the terms of the Creative Commons Attribution License 4.0, which permits unrestricted use, distribution, and reproduction in any medium, provided the original work is properly cited. 


\section{MATEC Web of Conferences}

two unknown variables, and the corresponding equation [5] are shown as follows:

$$
\begin{aligned}
& \frac{\partial(\rho k)}{\partial t}+\frac{\partial\left(\rho k u_{i}\right)}{\partial x_{i}}= \\
& \frac{\partial}{\partial x_{j}}\left[\left(\mu+\frac{\mu_{j}}{\sigma_{k}}\right) \frac{\partial k}{\partial x_{j}}\right]+G_{k}+G_{b}-\rho \varepsilon-Y_{M}+S_{k}
\end{aligned}
$$

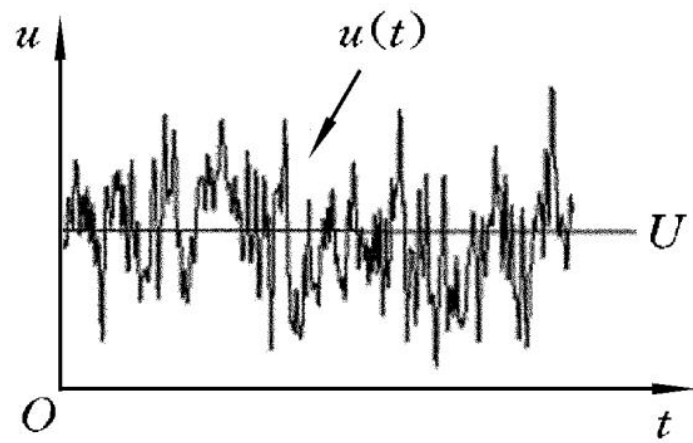

Figure 1. Speed of turbulence

$$
\begin{aligned}
& \frac{\partial(\rho \varepsilon)}{\partial t}+\frac{\partial\left(\rho \varepsilon u_{i}\right)}{\partial x_{i}} \\
& =\frac{\partial}{\partial x_{j}}\left[\left(\mu+\frac{\mu_{j}}{\sigma_{\varepsilon}}\right) \frac{\partial \varepsilon}{\partial x_{j}}\right]+C_{1 \varepsilon} \frac{\varepsilon}{k}\left(G_{k}+G_{3 \varepsilon} G_{b}\right) \\
& -C_{2 \varepsilon} \rho \frac{\varepsilon^{2}}{k}+S_{\varepsilon}
\end{aligned}
$$

in which, $G_{k}$ is generated by turbulence kinetic energy $k$ from mean speed gradient; $G_{b}$ is generated by $k$ from buoyant force, representing the contribution of pulsation extension in condensable flow; $C_{1 \varepsilon}, C_{2 \varepsilon}$ and $C_{3 \varepsilon}$ are respectively the constant by experience, the Prandt1 coefficient corresponding to and dissipation rate, and the source item which is defined by users.

To disperse the control differential equations, the finite volume method is used to decompose the differential equations into a series of multi-variable nonlinear coupling algebra equation set, and the one-order windward format is used to disperse the convection item, the second-order accuracy central differential format is used to disperse the extension item, and for pressure-speed coupling equation, the SIMPLE algorithm is used to solve the simultaneous equation.

\subsection{Grid model}

The real satcom antenna structure is very complex and contains a lot of components. To construct the model, the components that have little influence can be ignored. So the simplification is made on the radar reflector, the antenna beam, and the platform and rota- tion frame base, with the main features remained to make the computational results accord with the real situation. The simplification model is shown as Figure 2:

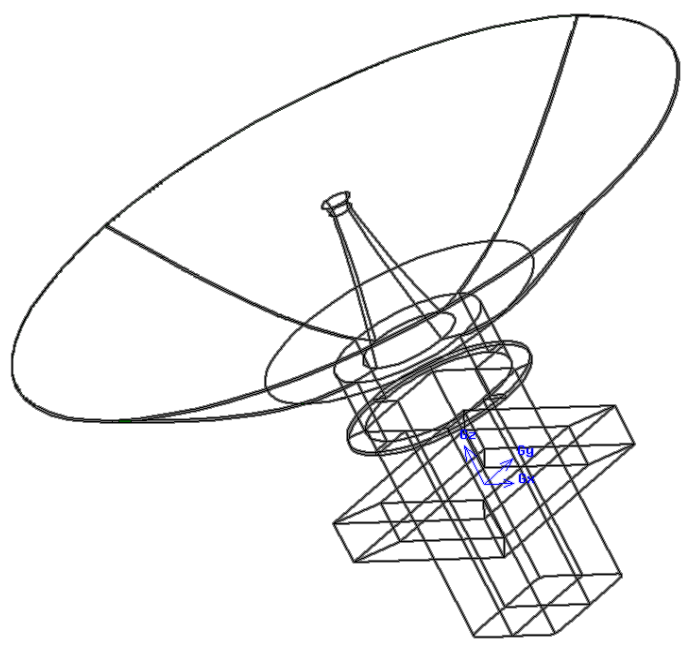

Figure 2. Model of antenna

The grid topology is a very important prerequisite to proceed flow field computation. The quality of grid division directly affects the convergence of the numerical computation. During the grid division, the first step is to divide the model into several parts. The cooper grid and Map grid can be used to proceed the grid division of the model. The second step is to generate the boundary layer, the near-wall layer and the computational region grid as shown in Figure 3 and Figure 4. The total number of grid is about 1,650,000.

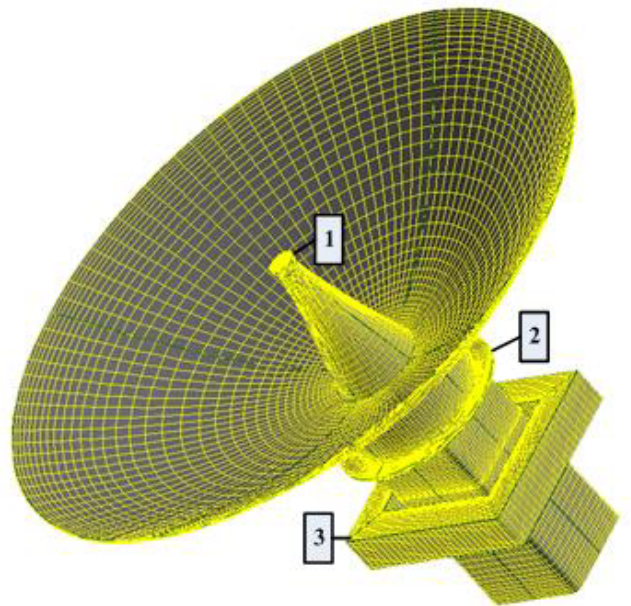

Figure 3. Boundary layer grid

\subsection{Computation result}

Generally speaking, initial condition has great influ- 
ence on the result. Thus, the given initial condition should be practical. In this paper, the remote field flow is used to initialize the global field. The process of CFD is favoring. The final residual error curve tends to be stable and the minimum error converges smaller than $10^{-5}$. The error of in-out flow difference is smaller than $0.5 \%$, and the result can be taken as convergence.

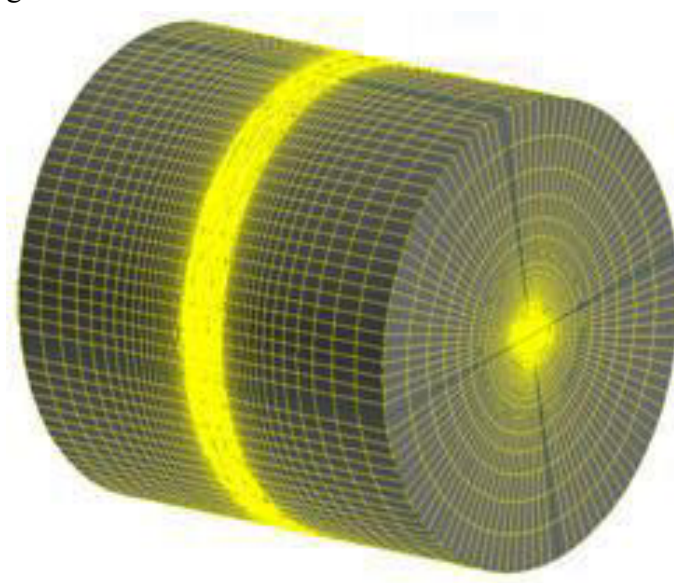

Figure 4. Computational region grid

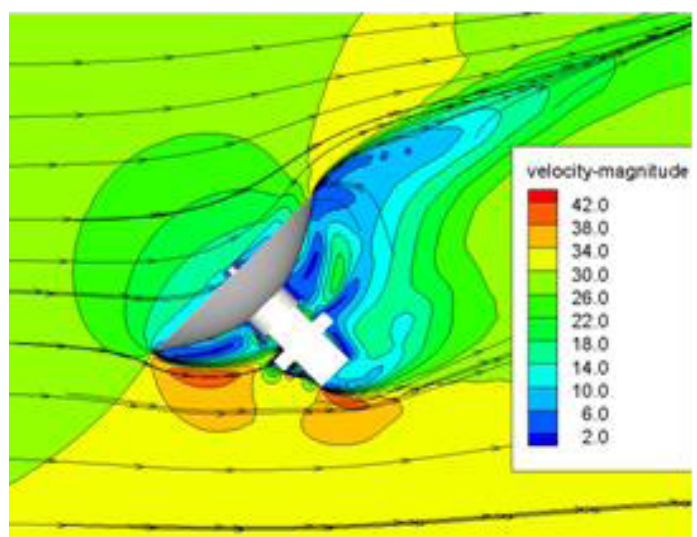

Figure 5. Speed distribution of antenna

The range of wind speed is chosen as $10-30 \mathrm{~m} / \mathrm{s}$, the range of pitch is chosen as $5^{\circ}-90^{\circ}$ and the range of windward angle is chosen as $0^{\circ}-180^{\circ}$. Six samples are chosen from wind speed, which are 10, 14, 18, 22, 26, and 30. Eight samples are chosen from pitch, which are $5,10,15,30,45,60,75$, and 90 . Eleven samples are chosen from windward angle, which are $0,20,40$, $60,80,90,100,120,140,160$, and 180 . Finally, there are 528 samples in total. From each sample, the pressure distribution of antenna reflector, the antenna flow field and three shafts data are acquired. And 140G data of flow field, 3168 figures and 1548 torque are acquired. When the wind speed of the computational result is $30 \mathrm{~m} / \mathrm{s}$, the pitch of antenna is $45^{\circ}$ and the windward angle is $40^{\circ}$. They are shown in Figure 5 and Figure 6:

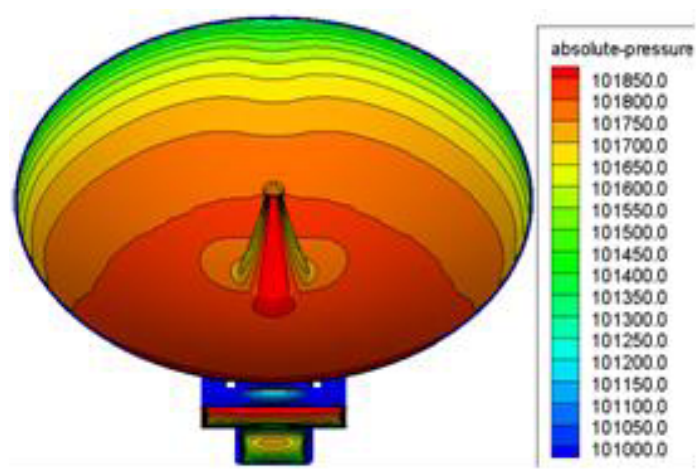

Figure 6. Pressure distribution of antenna

\section{SIMULATION OF ANTENNA SERVO SYSTEM}

The shipborne satcom antenna servo system uses the scheme of three-axis stabilization and two-axis tracking [6]. The three-axis includes the heading system, the pitch system and the crossing system. The three-axis stabilization is used to isolate the ship swing disturbance and stay stabilization by rotating the three-system to adjust the attitude of the antenna. The two-axis tracking is used to point out the target satellite in real-time by rotating heading system and pitch system to adjust the pointing angle of the antenna.

\subsection{Dynamics of the servo system}

To design the control system of the servo system, the control process should be described by math, which means to get the dynamics of the control object. The servo system rotates the antenna through the reduction gear box by controlling the drive power amplifier to output current to drive the motors. To analyze the servo system, the minor factors are ignored and the main factors are emphasized. The actuator and its transfer function of the servo system is the most important part during modeling. Because the shipborne satcom antenna servo system is very big, the resonance oscillation feature of the structure should be considered. All mass of the antenna is concentrated into one point and the rotational inertia is $J_{L}$. Concentrate the stiffness to two gear box, the equivalent stiffness of each gear box output shaft is $K_{L}$. Meanwhile, ignore the nonlinear factors such as gap and friction between gears, and the simplified physical model of the dual-motor drive servo system is acquired as shown in Figure 7.

$R_{a}$ is the sum of resistance in the motor armature circuit circle; $U_{a}$ is the voltage of motor armature; $L_{a}$ is the sum of inductance in the motor armature circle; $U_{e}$ is the counter electromotive force of motor; 


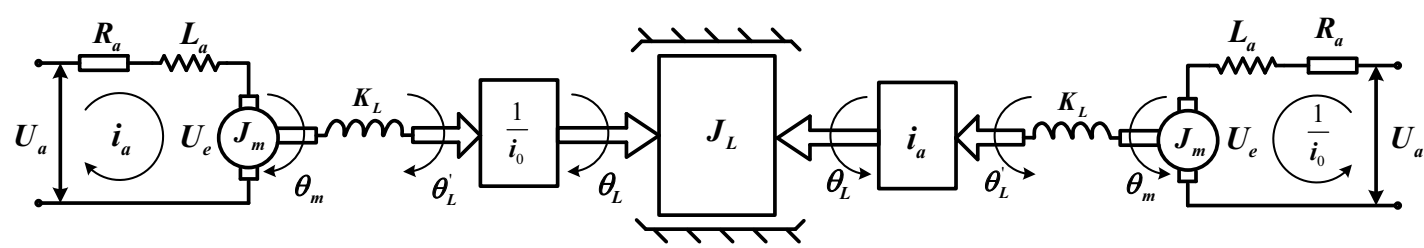

Figure 7. Physical model of the dual-motor drive servo system $i_{a}$ is the current in the circuit; $J_{m}$ is the rotational inertia of motor; $J_{L}$ is the rotational inertia of load; $\theta_{m}$ is angular displacement of motor; $\theta^{\prime}$ is the output angular displacement of the drive system; $\theta_{L}$ is the angular displacement of antenna and $i_{0}$ is the reduction ratio of gear box.

Based on the circuit voltage balance principle and Newton's law, we can obtain the following equations which are used to describe the circuit voltage and load balance respectively $[7,8]$.

$U_{a}=U_{e}+R_{a} i_{a}+L_{a} \frac{d i_{a}}{d t}$

$2 J_{m} \ddot{\theta}_{m}+2 K_{L}\left(\theta_{m}-\theta_{L}^{\prime}\right)+2 F_{m} \dot{\theta}_{m}=2 C_{m} i_{a}$

Where,

$$
\left\{\begin{array}{l}
U_{e}=C_{e} \dot{\theta}_{m} \\
M_{a}=C_{m} i_{a} \\
2 K_{L}\left(\theta_{m}-\theta_{L}^{\prime}\right)=J_{L}^{\prime} \ddot{\theta}_{L}^{\prime}+F_{L}^{\prime} \dot{\theta}_{L}^{\prime} \\
J_{L}^{\prime}=\frac{J_{L}}{i_{0}^{2}} \\
F_{L}^{\prime}=\frac{F_{L}}{i_{0}^{2}} \\
\theta_{L}^{\prime}=i_{0} \theta_{L}
\end{array}\right.
$$$$
\frac{\dot{\theta}_{m}}{U_{a}}=\frac{2 C_{m} K_{T}\left[\left(\frac{1}{\omega_{L}}\right)^{2} s^{2}+2 \xi_{L} \frac{1}{\omega_{L}} s+1\right]}{\left(L_{a} s+R_{a}\right)\left(1+T_{0} s\right)\left[\left(\frac{1}{\omega_{e}}\right)^{2} s^{2}+2 \xi_{e} \frac{1}{\omega_{e}} s+1\right]}
$$

$$
\frac{\dot{\theta}_{L}}{U_{a}}=\frac{2 C_{m} K_{T}}{i_{0}\left(L_{a} s+R_{a}\right)\left(1+T_{0} s\right)\left[\left(\frac{1}{\omega_{e}}\right)^{2} s^{2}+2 \xi_{e} \frac{1}{\omega_{e}} s+1\right]}
$$$$
\frac{\theta_{L}}{U_{a}}=\frac{2 C_{m} K_{T}}{i_{0}\left(L_{a} s+R_{a}\right)\left(1+T_{0} s\right)\left[\left(\frac{1}{\omega_{e}}\right)^{2} s^{2}+2 \xi_{e} \frac{1}{\omega_{e}} s+1\right] s}
$$

Where $C_{e}$ is the motor torque factor; $M_{a}$ is the output torque of motor; $F_{m}, F^{\prime}, F_{L}$ are respectively the viscosity factors of motor, the drive system and the antenna.

Because the change rate of current is far faster than angular rate, we can ignore the counter electromotive force, then $U_{e}=0$. And we can obtain as follows:

$$
\frac{M_{a}}{U_{a}}=\frac{C_{m}}{L_{a} s+R_{a}}
$$

Then we can obtain the transfer function of the system as follows:

Where, $\quad \omega_{L}=\sqrt{\frac{K_{L}}{J_{L}^{\prime}}} \quad, \quad \xi_{L}=\frac{1}{2} \frac{F_{L}^{\prime}}{\sqrt{K_{L}^{\prime} J_{L}^{\prime}}}$

$T_{0}=\frac{2 J_{m}+J_{L}^{\prime}}{2 F_{m}+F_{L}^{\prime}} \quad, \quad \omega_{e}=\sqrt{\frac{K_{L}\left(2 J_{m}+J_{L}^{\prime}\right)}{J_{m} J_{L}^{\prime}}}$

$\xi_{e}=\frac{1}{2} \times \frac{F_{m} F_{L}^{\prime}}{F_{m}+F_{L}^{\prime}} \times \sqrt{\frac{J_{m}+J_{L}^{\prime}}{K_{L}+J_{m} J_{L}^{\prime}}}, \quad K_{T}=\frac{1}{F_{m}+F_{L}^{\prime}}$

\subsection{System simulation}

Based on the analysis and modeling of the servo system, the simulation model is built in the MATLAB/Simulink environment to analyze the system. The parameters in the transfer function can be acquired from the practical satcom $[9,10]$.

First, the dynamic response of the actuator, the drive system and the load are analyzed. The step signal is imposed on the system to check the angle and angular rate of the antenna. The results are shown in Figure 8 and Figure 9.

From the results, the actuator, the drive system and the load have good dynamic response with fast response. The small overshoot and the system adjustment time are very short, which prove the accuracy of the dynamics to a certain extent. 
ICETA 2015

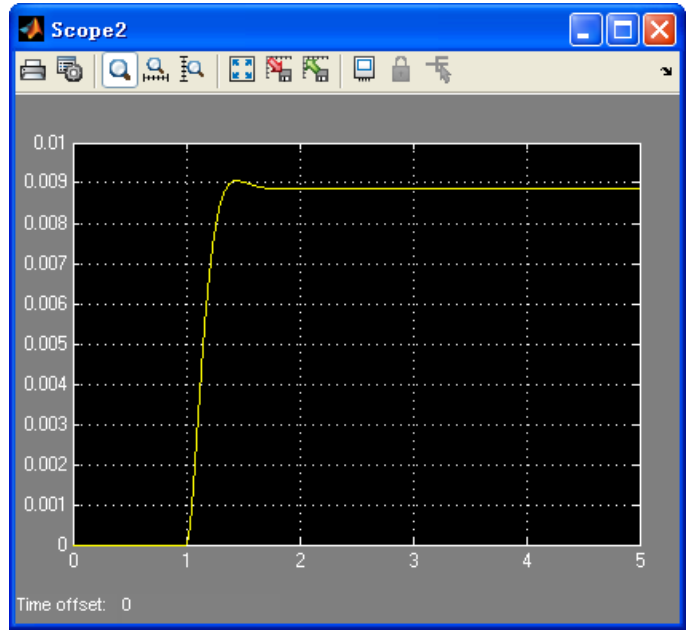

Figure 8. System dynamic response on step signal

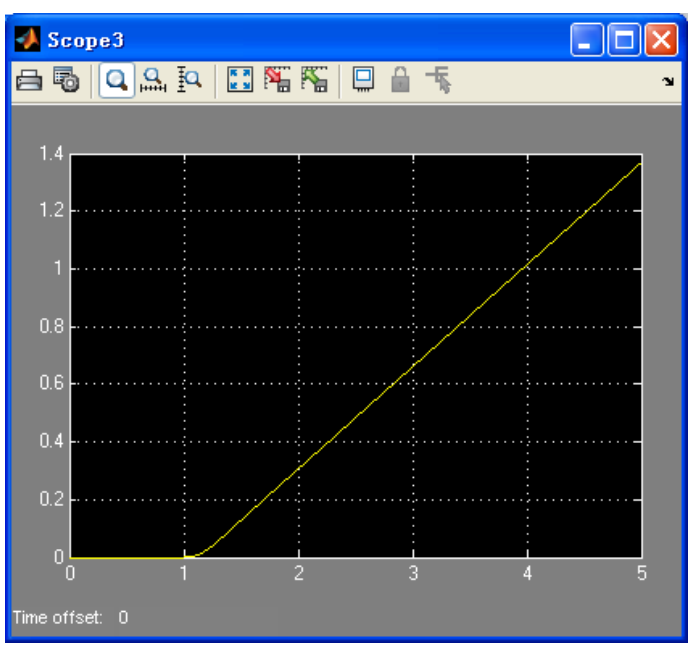

Figure 9. Angle output of the system

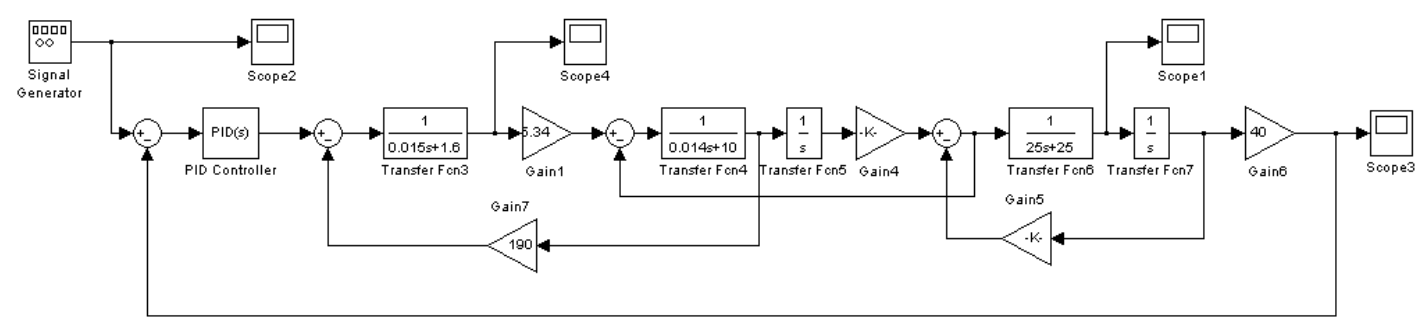

Figure 10. Simulation model of the antenna servo system

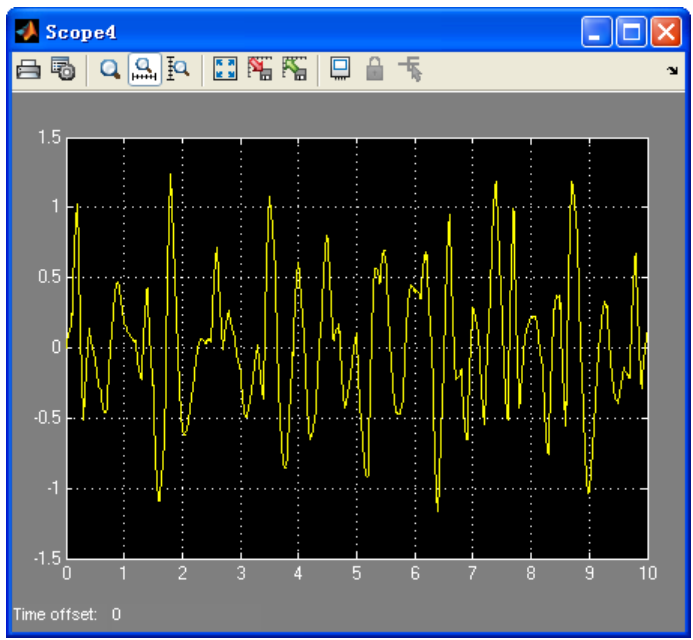

Figure 11. Predicting result of the drive current

The simulation model of the antenna servo system in MATLAB/Simulink is shown in Figure 10.

The signal generator is used to generate the ship swing signal. The controller is based on PID algorithm Under random ship swing, the drive current of the system from simulation is shown in Figure 11 and

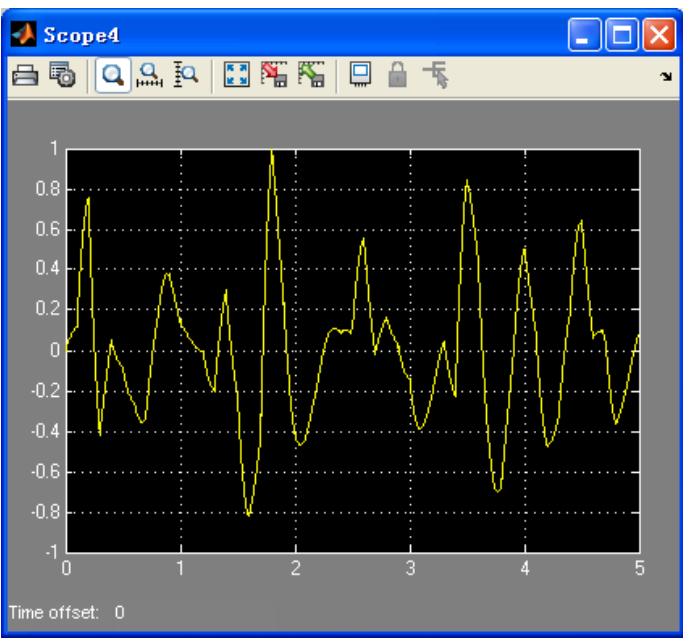

Figure 12. Drive current by simulation under random ship swing

Figure 12.

\subsection{Test and verification}

The practical drive current of the system during normal operation is shown in Table 1: 


\section{MATEC Web of Conferences}

Table 1. Drive current of the system during normal operation

\begin{tabular}{ll}
\hline \hline Parameter & Value \\
\hline Ship heading & $128.05^{\circ}$ \\
Wind speed & $16.9^{\circ}$ \\
Wind angle & $342^{\circ}$ \\
Heading & $147.84^{\circ}$ \\
Pitch & $36.15^{\circ}$ \\
Crossing & $0.44^{\circ}$ \\
Motor A & $-2.0 / 2.4$ \\
Motor E & $-2.9 / 1.8$ \\
Motor C & $-1.6 / 1.6$ \\
\hline \hline
\end{tabular}

Based on the parameters of three-axis torque in section 2 , by using ternary three-order interpolation algorithm, the equivalent torque of the state in Table 1 at the pitch motor (E motor) is acquired, which is $1027.96 \mathrm{Nm}$. By simulation, the drive current of the system is shown in Figure 12. The output drive current of pitch motor which is in accord with practical records (The practical result is the sum of current of $E$ motor 1 and motor 2 , which is 1.1 ) is near 1 . This comparison shows that the predicting result is quite well.

\section{CONCLUSIONS}

In this paper, the $k-\varepsilon$ model is used, and the division strategy of satcom antenna grid model, the generation method of boundary layer, the near-wall layer and the computational region grid are studied. And the suitable grid division method for the research in this paper is proposed. The wind load, the antenna and the three-axis torque are under different wind speed, the windward angle and pitch are calculated. The dynamics of antenna servo system and the simulation model are built under MATLAB/Simulink simulation environment. And the drive current under the simulate ship swing and wind load is acquired. Finally, the drive current predicting method according to wind speed, windward angle and antenna attitude is concluded and verification is made by comparison simulation results with practical records.

\section{REFERENCES}

[1] Selvem P R, 1997. Computation of pressures on Texas Tech University building using large eddy simulation, Journal of Wind Engineering and Industrial Aerodynamics.

[2] Du Q., \& Du P., 2010. University Analysis for Characteristics of Wind Loads on Antennas in Atmospheric Boundary Layer, Journal of University of Electronic Science, 39(2).

[3] DU Q., \& Du P., 2011. Large Eddy Simulation of Wind Loads on Phased Array Antennas Based on Synthetic Turbulent Inflow. Journal of Mechanical Engineering Jun, 47(12)
[4] Su M., \& Huang S., The Basic of Computational Fluid Dynamics, Beijing: Tsinghua Publishing, Inc.

[5] Fluent Inc. 2003. FLUENT User's Guide. Fluent Inc.

[6] Xin Y., 2009. Ship roll stabilization technology of space surveillance and control communications equipment, Beijing: National Defense Industry Press.

[7] Li L., \& Wang J., 1995. Dyanmics of gear system, Beijing: Science Press, pp. 105-110.

[8] Zhang L., \& Liao X., 2013. Modeling and control algorithm design of large-diameter antenna servo system, Journal of South China University of Technology (Natural Science Edition), 5: 22-27.

[9] Sun X., 2013. Design and Implementation of the Servo Control System of the Shipborne Communication in Moving, Xidian University.

[10]Jiang J., 2012. Research on Several Key Problems of Mobile Satellite Communication System Used on Moving Carrier, Nanjing University of Science and Technology. 